M 166 Köpf Märendichtung

M 167 Ebert Historische Syntax d. Deutschen

M 168 Bernstein Literatur d. deutschen Frühbumanismus

M 169 Leibfried/Werle Texte $z$. Theorie d. Fabel

M 170 Hoffmeister Deutsche u. europ. Romantik

M 171 Peter Friedricb Scblegel

M 172 Würffel Das deutsche Hörspiel

M 173 Petersen Max Frisch

M 174 Wilke Zeitschriften des 18. Jabrbunderts I: Grundlegung

M 175 Wilke Zeitscbriften des 18. Jabrbunderts II: Repertorium

M 176 Hausmann François Rabelais

M 177 Schlütter Das Sonett

M 178 Paul August Strindberg

M 179 Neuhaus Günter Grass

M 180 Barnouw Elias Canetti

M 181 Kröll Gruppe 47

M 182 Helferich G.W.Fr. Hegel

M 183 Schwenger Literaturproduktion

M 184 Naumann Literaturtheorie u. Geschichtsphilosophie, Teil I

M 185 Paulin Ludwig Tieck

M 186 Naumann Adalbert Stifter

M 187 Ollig Der Neukantianismus

M 188 Asmuth Dramenanalyse

M 189 Haupt Heinrich Mann

M 190 Zima Textsoziologie

M 191 Nusser Der Kriminalroman

M 192 Weißert Ballade

M 193 Wolf Martin Luther

J. B. METZLER 


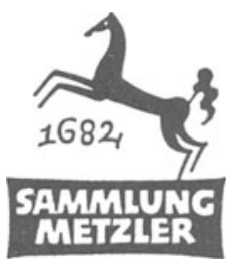

REALIEN ZUR LITERATUR ABT. D:

LITERATURGESCHICHTE 
JURGEN HAUPT

\section{Heinrich Mann}

MCMLXXX

J. B. METZLERSCHE VERLAGSBUCHHANDLUNG STUTTGART 
CIP-Kurztitelaufnahme der Deutschen Bibliothek

Haupt, Jürgen:

Heinrich Mann / Jürgen Haupt. - Stuttgart: Metzler 1980.

(Sammlung Metzler; M 189: Abt. D, Literaturgeschichte)

ISBN 978-3-476-10189-1

ISBN 978-3-476-10189-1

ISBN 978-3-476-03894-4 (eBook)

DOI 10.1007/978-3-476-03894-4

\section{189}

(c) Springer-Verlag GmbH Deutschland 1980

Ursprünglich erschienen bei J. B. Metzlersche Verlagsbuchhandlung und Carl Ernst Poeschel Verlag GmbH in Stuttgart 1980 


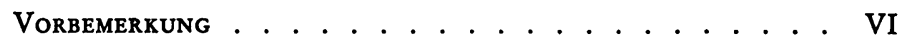

WERK-UBERSICHT $\ldots \ldots \ldots \ldots \ldots \ldots$

I. Materialien . . . . . . . . . . . . . 1

1. Bibliographien ............. 1

2. Gesamtausgaben . . . . . . . . . . 2

3. Briefe . . . . . . . . . . . . 4

4. Sonstige Texte und Dokumente . . . . . . . . . . . 7

5. Wichtige Gesamtdarstellungen . . . . . . . . . . . . 8

II. LEBEN UND WeRK . . . . . . . . . . . . . . . . . 12

1. Frühe Phase 1871-1904 Konservatismus und Ästhetizismus 12

a) 1871-1896/97 Kindheit, Entwicklung - Neuromantisches Jugendwerk . . . . . . 12

b) 1897-1904 Frühwerk: Künstler- und Bürger-Kritik 27

2. Mittlere Phase 1904-1933 Republikanismus und Moralismus . . . . . . . . . . . . . . 43

a) 1904-1910 Ubergang zum Republikanismus . . . 43

b) 1910-1923/24 Radikal-Republikanismus . . . . . . 59

c) 1923/24-1933 Kapitalismus- und Republik-Kritik . . 94

3. Späte Phase (Exil) 1933-1950 Sozialismus und Humanität . . . . . . . . . . . . . 132

a) 1933-1940 Antifaschistischer Kampf . . . . . . 132

b) 1940-1950 Alterswerk: Zeitalter-Kritik . . . . 170

III. Geschichte und Probleme der Forschung . . . . . . . 200

1. Geschichte der Wirkung, Rezeption, Forschung . . . . . 200

2. Forschungsprobleme . . . . . . . . . . . 207

a) Biographie, Periodisierung, Grundkategorien . . . 208

b) Das "Bruder-Problem « . . . . . . . . . . . . . . 210

c) Literarische Konstellationen . . . . . . . . . . . 214

d) Stil, Sprache, literarische Entwicklung . . . . . . 215

e) Literatur-Konzeption und literarische Gattungen . . 217

IV. Personenregister . . . . . . . . . . . . . . . . . . 221 


\section{VORBEMERKUNG}

"Soviel an mir lag, erreichte ich alles Wünschenswerte bei Personen, die ohnedies meiner Meinung waren. Selten überzeugen wir andere« ("Zeitalter", S. 263) "Das ist sehr schwer: Wahrheiten sagen und doch nicht ganz entmutigen " $(\mathrm{Br}$. a. K. Pinkus, 11. 2. 1938)

Eine Darstellung von "Leben und Werk « Heinrich Manns im geschichtlichen Kontext seiner und unserer Zeit (iim Lichte unserer Erfahrung') hängt von unseren Gesichtspunkten und Wertungen ab. Die gegenwärtig zunehmende Bedeutung Heinrich Manns als einer der wenigen großen >politischen Schriftsteller Deutschlands wird zur Geltung zu bringen gesucht durch eine politisch-ideologisch einheitlich vorgenommene $\mathrm{Pe}-$ riodisierung und eine entsprechend akzentuierte Darstellung seiner Entwicklung.

Heinrich Manns frühe, zunächst ästhetische, insgeheim bereits moralische, schließlich dezidiert politische Kritik am deutschen Bürgertum entwickelt sich im ersten Jahrzehnt nach $1900 \mathrm{zu}$ einem radikal republikanischen Bewußtsein. Wichtig ist das Jahr 1910: durch die berühmten, an der französischen Aufklärung und Revolution orientierten und den politischen Expressionismus vorbereitenden Manifeste von »Geist und Tat «. Weder der Beginn noch das Ende des Ersten Weltkrieges stellen einen tiefen Einschnitt in Heinrich Manns politischem Bewußtsein dar: Der imperialistische Krieg war für ihn voraussehbare Konsequenz der geschichtlich-gesellschaftlichen Entwicklungen. Wohl aber stellt das Jahr 1923, das Jahr der Inflation, Republik-Erschütterung und allgemeiner bürgerlichen Verunsicherung einen Einschnitt dar, der in seiner tiefgreifenden Wirkung auf Heinrich Mann m. E. bisher nicht genügend erkannt worden ist: Von den Erfahrungen des Jahres 1923 aus überprüft er sein bisheriges idealistisches Denk-System, gelangt zu immer schärferer, genauerer Bürgertums- und Kapitalismus-Kritik und zu tiefer Skepsis auch gegenüber der Weimarer Republik - deren kultureller Repräsentant er doch gleichwohl bleibt. Die Periodisierung der "späten Phase (1933-1950) eines idealistisch-ethischen Sozialismus ist in der Forschung - im Unterschied zur "frühen « und "mittleren « Phase - unumstrit- 
ten: eindeutig markiert durch die beiden unterschiedlichen Exil-Stationen in Frankreich und Amerika.

Ein zweites, hier bereits anzusprechendes Problem ist die Bewertung und Gewichtung des literarischen und des essayistischtheoretischen Werkes. Heinrich Mann gilt bis heute weithin als 'Romancier<; von seinen zwanzig Romanen - kein Jahr ohne Reflexion über und für Romane! - sind allerdings nur die wichtigen Wilhelminismus-Romane (»Unrat«, "Untertan«) und die späteren "Henri-Quatre«-Romane einigermaßen bekannt; die ehemals berühmten sfrühen< Romane, die WeimarerRepublik-Romane und die Altersromane werden vergleichsweise wenig gelesen (vgl. Werk-Übersicht).

Die zahlreichen Novellen scheinen für Heinrich Mann eher Nebenprodukte - quasi Roman-Kurzfassungen, -Variationen, -Episoden - gewesen zu sein. Die wenigen, heute vergessenen Dramen und Komödien sind vom Autor selber nur zum Teil wichtig genommen worden. Im übrigen sind Novellistik und Dramatik bereits Ende der Zwanziger Jahre so gut wie beendet. Demgegenüber tritt mehr und mehr der Essayist und $\mathrm{Pu}-$ blizist Heinrich Mann hervor - auch für den zunehmend größer werdenden Heinrich-Mann-Interessentenkreis seit Anfang der Siebziger Jahre in der Bundesrepublik. Die Fülle der politischen, kulturpolitischen und literarischen Essays, Schriften und Reden, ihr Rang neben den Romanen und ihre grundlegende theoriebildende Funktion für das belletristische Schaffen sind besonders zu berücksichtigen, wenn man heute, in einer sich abzeichnenden, vornehmlich dem politischen Autor geltenden Heinrich-Mann Wiederentdeckung sein 'Leben und Werk erfassen will.

Methodisch werden >Leben tung dargestellt, im Sinne Heinrich Manns selber: "Die Werke folgen als Ergebnis des Lebens, kann man sagen" (Br. a. K. Lemke, 30. 4. 1948).

Kein Gesamtwerk eines der großen Schriftsteller des 20. Jh.s liegt derart zersplittert, unvollständig und unübersichtlich in diversen Auswahl- und Einzel-Ausgaben vor wie dieses. Daher werden hier gerade auch die zahlreichen schwer oder nicht zugänglichen Werke relativ ausführlich dargestellt und Heinrich Manns Entwicklungsgang mit Zitaten aus kaum veröffentlichten oder schwierig auffindbaren Briefen, Essays, Dokumenten, Beurteilungen verständlich gemacht. (Wenn irgend möglich, wurde aus gegenwärtig in West-Deutschland greifbaren Texten 
zitiert.) Die Vielzahl der (zitierten) Stellungnahmen Heinrich Manns zu sich und zur Umwelt ist gleichfalls notwendig, denn sein Leben und Werk sind stets und intensiv auf Offentlichkeit bezogen gewesen. "Erfolg " und mehr: "Wirkung " sind Leitvorstellungen dieses Autors für seine Literatur und seine Politik - und daher auch in dieser Gesamt-Darstellung über ihn.

Herzlich danken möchte ich Elke Krause, Hannover, für die wesentliche Hilfe bei der Zusammenstellung und Kontrolle der bibliographischen Angaben. 


\section{WERK-ÜBERSICHT}

Schaffens-Phasen, wichtigste Werke (Entstehung)

ROMANE (gesamt)

1. 1892 In einer Familie (1892/93)

Romane der *Dekadenz*

Im Schlaraffenland

(1898/1900)

Die Göttinnen (1899/1902)

Die Jagd nach Liebe (1903)
NOVELLEN (Ausw.)/ SCHAUSPIELE (Sch.)

Das Wunderbare und and. Novellen (1894-97)

Ein Verbrechen $u$. and. Geschichten (1897/98)

Pippo Spano (1903)

ESSAYS (Ausw./Slg.)

Rezensionen

Kulturpol. Aufsätze im "Zwanzigsten Jahrhundert * (1895/96)

Flaubert-Sand (1905)

Abdankung (1905)

Flöten und Dolche

(Slg.) (1905)

Zwischen den Rassen

Die Branzilla (1906)

Die kleine Stadt (1907/09)

1910 * Romane des Kaiserreichs *

Der Untertan ([1907]-1914)

Die Armen (1916/17)

Schauspielerin (Sch.)

(1911)

Madame Legros (Sch.)

(1913)

Kobes (Sch.) (1923)

Voltaire-Goethe (1910)

Geist und Tat (1910)

Zola (1915)

Kaiserreich und Republik

(1919)

Die Tragödie von 1923

1923 * Romane der Republik*

Mutter Marie (1925/26)

Eugenie oder die Bürgerzeit (1927/28)

Die große Sache (1929/30)

Ein ernstes Leben

(1931/32)

Liliane und Paul (1925)

Suturp (1926)

Bibi (Kom.) (1928)

Deutsche Republik (1927)

Sieben Jahre (Slg.) (1929)

Das öffentl. Leben (Slg.)

(1932)

Bekenntnis zum Ubernationalen (1932)

Der $\mathrm{Haß}$ (Slg.) (1933)

Es kommt der Tag (Slg.)

(1936)

Der Weg der deutschen

Arbeiter (1936)

Mut (Slg.) (1939)

1940 "Altersromane*

Lidice (1942)

Eine Liebesgeschichte

(1943)

Ein Zeitalter wird besichtigt (1941/44) Der König von Preußen (1948)

Die traurige Geschichte von Friedrich dem Großen (1940-[1948])

Empfang bei der Welt (1941/45)

Der Atem (1946/47) 
Akz

Arbeitsh.

Arnold

AW

Banuls

BT

BZ

Dittb.

Dok.

Ebersbach

Ess. I; II; III

FAZ

FZ

GW

Hahn

Herden

HMA

Ihering

König
Akzente. Zeitschrift für Literatur. Hrsg. v. Hans Bender.

Arbeitshefte der Deutschen Akademie der Künste zu Berlin: Heinrich Mann am Wendepunkt der deutschen Geschichte. Internationale Konferenz aus Anlaß des 100. Geburtstages von Heinrich Mann. März 1971.

Arnold, Heinz-Ludwig (Hrsg.): Deutsche Literatur im Exil 1933-1945. Bd. 1. Frankfurt 1974.

Heinrich Mann. Ausgewählte Werke in Einzelausgaben. Hrsg. i. A. der Deutschen Akademie der Künste zu Berlin v. Alfred Kantorowicz. 13. Bde. (Bd. 13 v. d. Deutschen Akademie der Künste zu Berlin; besorgt v. Heinz Kamnitzer 1962.) Berlin 1951-1962.

'André Banuls: Heinrich Mann. Stuttgart 1970.

Berliner Tageblatt

Berliner Zeitung

Hugo Dittberner: Heinrich Mann. Eine kritische Einführung in die Forschung. Frankfurt a. M. 1974.

Heinrich Mann 1871-1950. Werk und Leben in Dokumenten und Bildern. Berlin und Leipzig 1971. ${ }^{2} 1977$.

Volker Ebersbach: Heinrich Mann. Leben-WerkWirken. Leipzig (Reclam) 1978. Dass.: Frankfurt a. M. (Röderberg-Verlag) 1978.

$=\mathrm{AW}$ Bd. XI-XIII

Frankfurter Allgemeine Zeitung

Frankfurter Zeitung

Heinrich Mann. Gesammelte Werke. Hrsg. v. der Deutschen Akademie der Künste zu Berlin. (Ab 1972: hrsg. von der Akademie der Künste der DDR). Redaktion: Sigrid Anger.

Manfred Hahn: Das Werk Heinrich Manns. Von den Anfängen bis zum »Untertan « 1885-1914. Diss. Leipzig 1965.

(Résumé von Kap. 1-9 und überarbeitete Fassung von Kap. 10 der Diss. in: WB 12, 1966, S. 363-406)

Werner Herden: Geist und Macht. Heinrich Manns Weg an die Seite der Arbeiterklasse. Berlin und Weimar 1971.

Heinrich-Mann-Archiv. Berlin-Ost.

Herbert Ihering: Heinrich Mann. Sein Werk und sein Leben. Berlin (Aufbau) 1952.

Hanno König: Heinrich Mann. Dichter und Moralist. Tübingen 1972. 
Matthias

Mitt.

$\mathrm{NDH}$

NDL

NTB

NWB

SuF

Schröter I

Schröter II

Schröter III

Text + Kritik

Verteidigung

Völker

Walter

WB

Werner I

Werner II

Weisstein I

Weisstein II

WZUJ

ZA

ZPSK
Heinrich Mann 1871-1971. Hrsg. v. Klaus Matthias. München 1973.

Mitteilungsblatt des Arbeitskreises Heinrich Mann. Hrsg. v. Siegfried Sudhof und Walter Biedermann in Zusammenarbeit mit dem Senat der Hansestadt Lübeck, Amt für Kultur. Seit Juli 1972 halbjährlich fortlaufend.

Neue Deutsche Hefte

Neue Deutsche Literatur

Das Neue Tage-Buch

Die Neue Weltbühne

Sinn und Form

Klaus Schröter: Anfänge Heinrich Manns. Zu den Grundlagen seines Gesamtwerkes. Stuttgart 1965.

Klaus Schröter: Heinrich Mann in Selbstzeugnissen und Bilddokumenten. rororo Monographien. Hamburg 1967.

Klaus Schröter: Heinrich Mann. »Untertan-ZeitalterWirkung«. Drei Aufsätze. Stuttgart 1971.

Heinz-Ludwig Arnold (Hrsg.): Text + Kritik. Sonderband Heinrich Mann. München 1971.

Heinrich Mann: Verteidigung der Kultur. Antifaschistische Streitschriften und Essays. Hrsg. v. Werner Herden. Berlin und Weimar (Aufbau) 1971.

Klaus Völker: Bertolt Brecht. Eine Biographie. München (Hanser) 1976.

Hans-Albert Walter: Deutsche Exilliteratur 19331950. Bd. 7. Darmstadt 1974.

Weimarer Beiträge

Renate Werner: Skeptizismus, Ästhetizismus, Aktivismus. Der frühe Heinrich Mann. Düsseldorf 1972.

Renate Werner (Hrsg.): Heinrich Mann. Texte zu seiner Wirkungsgeschichte in Deutschland. Tübingen 1977.

Ulrich Weisstein: Heinrich Mann. Eine historischkritische Einführung in sein dichterisches Werk. Mit einer Bibliographie der von ihm veröffentlichten Schriften. Tübingen 1962.

Ulrich Weisstein: Heinrich Mann in Amerika. In: Spalek, John u. a. (Hrsg.): Deutsche Exilliteratur seit 1933. Bd. 1 Kalifornien. Bern, München 1976. S. 442472.

Wissenschaftliche Zeitschrift der Friedrich-SchillerUniv. Jena.

Heinrich Mann: Ein Zeitalter wird besichtigt. Reinbek bei Hamburg (Rowohlt) 1976.

Zeitschrift für Phonetik, Sprachwissenschaft und Kommunikationsforschung. Berlin-Ost. 